\title{
The Order of Numerical Methods for Ordinary Differential Equations
}

\author{
By J. C. Butcher
}

\begin{abstract}
For a general class of methods, which includes linear multistep and RungeKutta methods as special cases, a concept of order relative to a given starting procedure is defined and an order of convergence theorem is proved. The definition is given an algebraic interpretation and illustrated by the derivation of a particular fourth-order method.
\end{abstract}

1. Introduction. Detailed theories have been published for the study of error propagation in linear multistep methods on the one hand ([1], [2], [3]) and for RungeKutta methods on the other hand ([2], [4]). While it is possible to modify these theories to include various methods which fall between these extreme types ([5], [6], [7], [8], [9]), it is of interest to analyse the behaviour of error propagation for a class of method of sufficient generality to include all these special cases in a natural and straightforward way. In fact, we will consider the type of general method formulated by the author [10].

In attempting to find a suitable meaning for the concept of "order" which generalizes the standard definitions in the well-known special cases, a rather surprising fact arises. This is that the definition of order which seems most natural for this general type of method does not necessarily, in the standard cases, assign a value to the order which coincides with that given by traditional definitions. In fact, this new concept coincides in the case of Runge-Kutta methods with "effective order" [11] so that, for example, there exist methods of order 5 in the sense of this paper but only 4 in the usual sense.

In the present paper, some of the ideas and methods introduced in [12] will be made use of to show how the analytic definition of order may be expressed in algebraic terms. To illustrate these developments an example is given of a method whose derivation is based on this new meaning of order.

2. Notation. Let $V$ be an $N$-dimensional real vector space and let $A, B$ be the matrices of two linear operators $V \rightarrow V$ with components $a_{i j}, b_{i j}$ respectively $(i, j=$ $1, \cdots, N)$. As in [10], we consider methods $(A, B)$ characterized by such a pair of matrices.

Let $X$ be a finite-dimensional normed real vector space and let $f: X \rightarrow X$ satisfy a Lipschitz condition with constant $L$. We shall consider the differential equation $y^{\prime}(x)=f(y(x))$. In computing solutions using $(A, B)$, we have, at the end of step number $n$, a collection of $N$ approximations $y_{1}^{(n)}, y_{2}^{(n)}, \cdots, y_{N}^{(n)}$ which are computed from

Received November 16, 1972.

AMS (MOS) subject classifications (1970). Primary 65L05; Secondary 34A50.

Key words and phrases. Initial-value problems, multistep methods, Runge-Kutta methods, order, convergence. 
corresponding approximations to the solution after $n-1$ steps. We write $h$ as the stepsize. That is, we regard $y_{i}^{(n)}(i=1,2, \cdots, N)$ as approximations at points $h$ further ahead than the approximations $y_{i}^{(n-1)}(i=1,2, \cdots, N)$. The formula for the computation of $y_{i}^{(n)}(i=1,2, \cdots, N)$ is

$$
y_{i}^{(n)}=\sum_{i=1}^{N} a_{i j} y_{i}^{(n-1)}+h \sum_{i=1}^{N} b_{i j} f\left(y_{j}^{(n)}\right) .
$$

To represent this equation more compactly, we make use of the vector space $V(X)$ defined as the direct product of $V$ and $X$. If $v=v_{1} \oplus v_{2} \oplus \cdots \oplus v_{N} \in V(X)$ where $v_{1}, v_{2}, \cdots, v_{N} \in X$, then we define $\|v\|=\max _{1 \leqq i \leqq N}\left\|v_{i}\right\|$. Given a linear operator $A: V \rightarrow V$, we define $[A]: V(X) \rightarrow V(X)$ by

$$
[A]\left(v_{1} \oplus v_{2} \oplus \cdots \oplus v_{N}\right)=\sum_{j=1}^{N} a_{1 j} v_{i} \oplus \sum_{i=1}^{N} a_{2 j} v_{j} \oplus \cdots \oplus \sum_{j=1}^{N} a_{N j} v_{j} .
$$

We thus write (2.1) in the more compact form used in [10]

$$
Y^{(n)}=[A] Y^{(n-1)}+h[B] F\left(Y^{(n)}\right)
$$

where $Y^{(n)}=y_{1}^{(n)} \oplus y_{2}^{(n)} \oplus \cdots \oplus y_{N}^{(n)}$ and the function $F: V(X) \rightarrow V(X)$ is defined by

$$
F\left(v_{1} \oplus v_{2} \oplus \cdots \oplus v_{N}\right)=f\left(v_{1}\right) \oplus f\left(v_{2}\right) \oplus \cdots \oplus f\left(v_{N}\right) .
$$

Throughout this paper, we will assume that the method $(A, B)$ is stable and consistent. That is, $\sup \left\{\left\|A^{n}\right\|: n=1,2, \cdots\right\}<\infty, A s=s$ and $A \xi+B s=\xi+s$ where $s \in V$ is defined by $s_{i}=1(i=1,2, \cdots, N)$ and $\xi$ is some member of $V$.

3. Definitions of Order. In framing our definition, we are motivated by a number of considerations. In the first place, we might consider the obvious generalization of the definition of order for multistep methods. This would lead to the following definition.

Definition 3.1. $(A, B)$ is of $M S$-order $q$ if there is $\xi \in V$ such that, for $k=$ $0,1, \cdots, q$ and $i=1,2, \cdots, N$,

$$
\xi_{i}^{k}=\sum_{j=1}^{N} a_{i j}\left(\xi_{i}-1\right)^{k}+k \sum_{j=1}^{N} b_{i j} \xi_{j}^{k-1} .
$$

Note that this definition in the case $q=1$ corresponds exactly to the definition of consistency.

If we applied Definition 3.1 as it stands to Runge-Kutta methods expressed in the form $(A, B)$, we would conclude that every explicit Runge-Kutta method has $M S$-order no greater than 1 . This is because the first off-step approximation found in a Runge-Kutta step uses only the approximation at the beginning of the step and, in fact, computes this new value by an Euler step. Later stages may make use of this first-order approximation to construct other approximations at various off-step points,but the final value given at the end of the step is designed to allow the effects of the lower order errors to cancel out. Of course, only this final approximation is used by subsequent steps. These considerations lead to the following definition.

Definition 3.2. $(A, B)$ is of $R K$-type if there is $I \in\{1,2, \cdots, N\}$ such that for all $v \in V$ and $i \in\{1,2, \cdots, N\}$ it holds that $(A v)_{i}=v_{I}$. In this case, we can reframe the method as an equivalent Runge-Kutta method and we define the $R K$-order as the order of that method. 
This definition, besides being applicable only to a limited class of methods, suffers from the disadvantage that it is not symmetrical in the members of $\{1,2, \cdots, N\}$. One way of combining features of these two definitions is to note that multistep methods in general require starting procedures. In the case of linear multistep methods, for example, Runge-Kutta methods are often used to supply the starting vectors $y_{1}^{(0)}, \cdots, y_{N}^{(0)}$. Furthermore, in the case of methods of $R K$-type, the internal computations in step $n$ could be regarded as being themselves computed from Runge-Kutta methods starting from $Y_{I}^{(n-1)}$.

Let $r$ denote a Runge-Kutta method. For a fixed function $f$, we interpret $r$ as a function on the real numbers which for stepsize $h$ gives a mapping $r(h): X \rightarrow X$ which takes $z \in X$ into the value computed using the Runge-Kutta method $r$ from starting value $z$. If we have a collection of Runge-Kutta methods $r_{1}, r_{2}, \cdots, r_{N}$, then we can construct a method which gives $N$ results $\bar{r}_{1}, \bar{r}_{2}, \cdots, \bar{r}_{N}$ given by

$$
\bar{r}_{i}(h)(z)=\sum_{i=1}^{N} a_{i j} r_{i}(h)(z)+h \sum_{j=1}^{N} b_{i i} f\left(\bar{r}_{j}(h)(z)\right) \text {. }
$$

Note that $\bar{r}_{1}, \bar{r}_{2}, \cdots, \bar{r}_{N}$ are Runge-Kutta methods also, so that we can interpret a method $(A, B)$ as a mapping on the class of $N$-tuples of Runge-Kutta methods to this same class.

For given $z \in X$, let $P_{h}(z)$ denote the value of $y(h)$ such that $y$ satisfies $y(0)=z$, $y^{\prime}(\xi)=f(y(\xi))$ for all $\xi$ in the open interval $\{\xi: \xi(h-\xi)>0\}$ and such that $y$ is continuous on the closed interval $\{\xi: \xi(h-\xi) \geqq 0\}$.

Definition 3.3. $(A, B)$ is of order $q$ relative to $r_{1}, r_{2}, \cdots, r_{N}$ if for all $f$ with continuous partial derivatives of order $q$ in a neighbourhood of $z$, it holds that

$$
\left\|\bar{r}_{i}(h)(z)-r_{i}(h)\left(P_{h}(z)\right)\right\|=O\left(|h|^{q+1}\right)
$$

as $|h| \rightarrow 0$ for $i=1,2, \cdots, N$.

Definition 3.4. $(A, B)$ is of order $q$ if there exist Runge-Kutta methods $r_{1}, r_{2}, \cdots, r_{N}$ such that $(A, B)$ is of order $q$ relative to $r_{1}, r_{2}, \cdots, r_{N}$.

To make practical use of this definition, we use algorithms consisting of three parts: (a) a starting procedure, (b) a continuing procedure, (c) a finishing procedure. Suppose the differential equation $y^{\prime}(\xi)=f(y(\xi))$ is given together with the value of $y\left(x_{0}\right)$. It is required to compute $y\left(x_{v}\right)$ where $x_{v}=x_{0}+\nu h$. The three parts of the method are as follows: (a) we compute $Y^{(0)}$ by $y_{i}^{(0)}=r_{i}(h)\left(y\left(x_{0}\right)\right)$ for $i=1,2, \cdots, N$, (b) we use the method $(A, B)$ to compute in turn $Y^{(1)}, Y^{(2)}, \cdots, Y^{(v)}$, (c) we choose one of $y_{1}^{(\nu)}, y_{2}^{(\nu)}, \cdots, y_{N}^{(\nu)}$, say $y_{I}^{(\nu)}$, and compute an approximation to $y\left(x_{\nu}\right)$ by the Runge-Kutta method (in general an implicit method) $r$ such that $r(h)=r_{I}(h)^{-1}$ (this inverse function exists for sufficiently small $h$ ) operating on $y_{I}^{(\nu)}$.

There are, of course, certain generalizations possible. For example, the finishing formula may be chosen in such a way that it not only reverses the effect of the perturbation to the initial value introduced by the starting formula but also carries the solution forward for a further step or part of a step. It is also possible, in some cases, to find simple finishing formulae which make use of more than one of $y_{1}^{(\nu)}, y_{2}^{(\nu)}, \cdots, y_{N}^{(\nu)}$.

4. Rate of Convergence. We consider a sequence of approximations to $y(x)$ given by the algorithm described in the previous section. That is, for $\nu=\nu_{0}, \nu_{0}+1$, $\cdots$, we use a value $h=\left(x-x_{0}\right) / \nu$ and apply that procedure. We obtain an approxi- 
mation for each such $\nu$ and we are interested in the behaviour of the error in these approximations as $h \rightarrow 0$ (that is, as $\nu \rightarrow \infty$ ).

Denote by $Y_{v}^{(n)}$ the value of $Y^{(n)}$ computed with stepsize $h=\left(x-x_{0}\right) / \nu$ and write $Y_{\nu}^{(n)}=y_{\nu, 1}^{(n)} \oplus y_{\nu, 2}^{(n)} \oplus \cdots \oplus y_{\nu, N}^{(n)}$. The initial values are given by $y_{\nu, i}^{(0)}=r_{i}(h)\left(y\left(x_{0}\right)\right)$ for $i=1,2, \cdots, N$ and the final approximation is given by $\tilde{y}_{\nu}=r_{I}(h)^{-1}\left(y_{\nu, I}^{(\nu)}\right)$.

THEOREM 4.1. If the method $(A, B)$ is of order $q$ relative to $r_{1}, r_{2}, \cdots, r_{N}$ and if all partial derivatives of $f$ of order up to $q$ are continuous and bounded in an open set in $X$ containing $Y=\left\{y(\xi):\left(\xi-x_{0}\right)(x-\xi) \geqq 0\right\}$, there exist constants $C, \nu_{0}$ such that, for $\nu \geqq \nu_{0}$,

$$
\left\|\tilde{y}_{\nu}-y(x)\right\| \leqq C \nu^{-a} .
$$

Proof. Let $H_{\nu}^{(n)}=\eta_{\nu, 1}^{(n)} \oplus \eta_{\nu, 2}^{(n)} \oplus \cdots \oplus \eta_{\nu, N}^{(n)} \in V(X)$ be defined by $\eta_{\nu, i}^{(n)}=r_{i}(h)\left(y\left(x_{0}+n h\right)\right)$ for $i=1,2, \cdots, N$. Also define $Z_{\nu}^{(n)}=H_{\nu}^{(n)}-y_{\nu}^{(n)}$ and $W_{\nu}^{(n)}=F\left(H_{\nu}^{(n)}\right)-F\left(Y_{\nu}^{(n)}\right)$ so that $\left\|W_{\nu}^{(n)}\right\| \leqq L\left\|Z_{\nu}^{(n)}\right\|$. Our first task is to estimate

$$
\begin{aligned}
E_{\nu}^{(n)} & =Z_{\nu}^{(n)}-[A] Z_{\nu}^{(n-1)}-h[B] W_{\nu}^{(n)} \\
& =H_{\nu}^{(n)}-[A] H_{\nu}^{(n-1)}-h[B] F\left(H_{\nu}^{(n)}\right) .
\end{aligned}
$$

We write $E_{\nu}^{(n)}=e_{\nu, 1}^{(n)} \oplus e_{\nu, 2}^{(n)} \oplus \cdots \oplus e_{\nu, N}^{(n)}$ and we find that

$$
\begin{aligned}
e_{\nu, i}^{(n)}= & r_{i}(h)\left(y\left(x_{0}+n h\right)\right)-\sum_{i=1}^{N} a_{i j} r_{i}(h)\left(y\left(x_{0}+(n-1) h\right)\right)-h \sum_{j=1}^{N} b_{i j} f\left(r_{j}(h)\left(x_{0}+n h\right)\right) \\
= & \left\{\bar{r}_{i}(h)\left(y\left(x_{0}+(n-1) h\right)\right)-\sum_{j=1}^{N} a_{i j} r_{j}(h)\left(y\left(x_{0}+(n-1) h\right)\right)\right. \\
& \left.-h \sum_{j=1}^{N} b_{i j} f\left(\bar{r}_{i}(h)\left(y\left(x_{0}+(n-1) h\right)\right)\right)\right\} \\
& +\left\{r_{i}(h)\left(y\left(x_{0}+n h\right)\right)-\bar{r}_{i}(h)\left(y\left(x_{0}+(n-1) h\right)\right)\right. \\
& \left.-h \sum_{i=1}^{N} b_{i j}\left(f\left(r_{j}(h)\left(y\left(x_{0}+n h\right)\right)\right)-f\left(\bar{r}_{i}(h)\left(y\left(x_{0}+(n-1) h\right)\right)\right)\right)\right\} \\
= & r_{i}(h)\left(P_{h}\left(y\left(x_{0}+(n-1) h\right)\right)\right)-\bar{r}_{i}(h)\left(y\left(x_{0}+(n-1) h\right)\right) \\
& +h \sum_{i=1}^{N} b_{i j}\left(f\left(\bar{r}_{i}(h)\left(y\left(x_{0}+(n-1) h\right)\right)\right)-f\left(r_{i}(h)\left(P_{h}\left(y\left(x_{0}+(n-1) h\right)\right)\right)\right) .\right.
\end{aligned}
$$

Because of the boundedness of the partial derivatives of $f$ at all points sufficiently close to the points in $Y$, we can, by requiring $h$ to be sufficiently small, obtain a uniform bound $C_{1}|h|^{a+1}$ for the difference occurring in (3.2) for all $z$ in $Y$ and all $i=1,2, \cdots, N$. We therefore have, for some $\nu_{1}$ and all $\nu \geqq \nu_{1}$, the following bound

$$
\left\|e_{\nu, i}^{(n)}\right\| \leqq C_{1}|h|^{a+1}(1+|h| L \| B||) \leqq C_{2}|h|^{a+1}
$$

where $C_{2}=C_{1}\left(1+L\|B\| \cdot\left|x-x_{0}\right| / \nu_{1}\right)$. Hence, $\left\|E_{\nu}^{(n)}\right\| \leqq C_{2}|h|^{a+1}$ for $\nu \geqq \nu_{1}$.

From (4.1) and the fact that $Z^{(0)}=0$, we obtain the equation

$$
Z_{\nu}^{(n)}=h \sum_{j=1}^{n}\left[A^{n-i} B\right] W_{\nu}^{(j)}+\sum_{j=1}^{n}\left[A^{n-i}\right] E_{\nu}^{(i)} .
$$


Let $\alpha, \beta$ be chosen so that $\left\|A^{i}\right\| \leqq \alpha,\left\|A^{i} B\right\| \leqq \beta$ for all $i=0,1,2, \cdots$. We see that $\left\|Z_{\nu}^{(n)}\right\| \leqq \epsilon_{\nu}^{(n)}$ where $\epsilon_{\nu}^{(0)}=0$ and

$$
\epsilon_{\nu}^{(n)}=|h| L \beta \sum_{j=1}^{n} \epsilon_{\nu}^{(j)}+n C_{2} \alpha|h|^{a+1}
$$

Hence,

$$
\epsilon_{\nu}^{(n)}-\epsilon_{\nu}^{(n-1)}=|h| L \beta \epsilon_{\nu}^{(n)}+C_{2} \alpha|h|^{a+1} .
$$

If $L \beta=0$, this leads to the equation

$$
\left\|z_{\nu}^{(\nu)}\right\| \leqq C_{3}|h|^{a},
$$

where $C_{3}=C_{2} \alpha\left|x-x_{0}\right|$ for all $\nu \geqq \nu_{2}=\nu_{1}$. Otherwise, we have

$$
(1-|h| L \beta)\left(\epsilon_{\nu}^{(n)}+|h|^{a} C_{2} \alpha / L \beta\right)=\left(\epsilon_{\nu}^{(n-1)}+|h|^{\alpha} C_{2} \alpha / L \beta\right) .
$$

In this case, we choose $\nu_{2}$ such that, if $h=\left(x-x_{0}\right) / \nu_{2}$, then $|h| L \beta<1$ and such that $\nu_{2} \geqq \nu_{1}$. We then find

$$
\epsilon_{\nu}^{(n)}+|h|^{q} C_{2} \alpha / L \beta=(1-|h| L \beta)^{-n}\left(\epsilon_{\nu}^{(0)}+|h|^{\alpha} C_{2} \alpha / L \beta\right)
$$

so that

$$
\epsilon_{\nu}^{(\nu)}=\left((1-|h| L \beta)^{-\nu}-1\right)|h|^{q} C_{2} \alpha / L \beta .
$$

In this case, we choose

$$
C_{3}=\frac{C_{2} \alpha}{L \beta}\left(\exp \left(\frac{\left|x-x_{0}\right| L \beta}{1-L \beta\left|x-x_{0}\right| / \nu_{2}}\right)-1\right)
$$

so that (4.2) holds in this case as well with $\nu \geqq \nu_{2}$. Having obtained this bound on $\left\|H_{v}^{(v)}-Y_{v}^{(v)}\right\|$, we must still estimate

$$
\begin{aligned}
\tilde{y}_{\nu}-y(x) & =r_{I}(h)^{-1}\left(y_{\nu, I}^{(\nu)}\right)-r_{I}(h)^{-1}\left(r_{I}(h)(y(x))\right) \\
& =r_{I}(h)^{-1}\left(y_{\nu, I}^{(\nu)}\right)-r_{I}(h)^{-1}\left(\eta_{\nu, I}^{(\nu)}\right) .
\end{aligned}
$$

Let the Runge-Kutta method $r_{I}(h)^{-1}$ be one that computes $s+1$ approximations $z_{0}, z_{1}, \cdots, z_{\mathrm{s}}$ from an initial value $z$ by the formula

$$
z_{i}=z+h \sum_{i=0}^{s} c_{i j} f\left(z_{j}\right), \quad i=0,1, \cdots, s,
$$

and gives a result $r_{I}(h)^{-1}(z)=z_{s}$. Since $r_{I}(h)^{-1}$ is, in general, implicit, we assume that $|h| L \max _{i} \sum_{i=0}^{s}\left|c_{i j}\right|<1$ to guarantee existence and uniqueness of $z_{0}, z_{1}, \cdots, z_{s}$. This is achieved by requiring that $\nu \geqq \nu_{3}$, where $\left|x-x_{0}\right| L \max _{i} \sum_{i=0}^{s}\left|c_{i j}\right| / \nu_{3}=k$ say, satisfies $k<1$. We now define $\nu_{0}$ as the greater of $\nu_{2}$ and $\nu_{3}$ and, from now on, we assume that $\nu \geqq \nu_{0}$. If $\bar{z} \in X$ and $\bar{z}_{0}, \bar{z}_{1}, \cdots, \bar{z}_{s}$ are computed from $\bar{z}$ in the same way as $z_{0}, z_{1}, \cdots, z_{s}$ are computed from $z$, we will now estimate $r_{I}(h)^{-1}(z)-r_{I}(h)^{-1}(\bar{z})$. From (4.3) and the corresponding equation for $\bar{z}_{0}, \bar{z}_{1}, \cdots, \bar{z}_{s}$, we have

$$
z_{i}-\bar{z}_{i}=z-\bar{z}+h \sum_{i=0}^{s} c_{i j}\left(f\left(z_{j}\right)-f\left(\bar{z}_{i}\right)\right)
$$


so that if $\max _{i}\left\|z_{i}-\bar{z}_{i}\right\|=\zeta$ then $\zeta \leqq\|z-\bar{z}\|+k \zeta$. Hence

$$
\left\|r_{I}(h)^{-1}(z)-r_{I}(h)^{-1}(\bar{z})\right\| \leqq(1-k)^{-1}\|z-\bar{z}\|
$$

so that if we write $C_{4}=C_{3} /(1-k)$, and substitute $z=y_{\nu, I}^{(v)}, \bar{z}=\eta_{\nu, I}^{(\nu)}$ and make use of the inequality $\left\|y_{\nu, I}^{(\nu)}-\eta_{\nu, I}^{(\nu)}\right\| \leqq\left\|Y_{\nu}^{(\nu)}-H_{\nu}^{(\nu)}\right\|$, then we find

$$
\left\|\tilde{y}_{\nu}-y(x)\right\| \leqq C_{4}|h|^{\alpha} .
$$

To obtain the result of the theorem, we write $C=C_{4}\left|x-x_{0}\right|^{q}$ and substitute $h=$ $\left(x-x_{0}\right) / \nu$ in $(4.4)$.

5. Algebraic Criterion for Order. In this section, we make use of the terminology of [12]. The basic idea in that paper is that there is a group $G$ whose elements may be represented by real-valued functions on the set $T$ of (rooted) trees and such that every member of a certain class of method (which includes all Runge-Kutta methods) can be characterised by a member of the group. In particular, $p$ is the group element associated with the exact increment function for a differential equation integrated through a unit step. If $m_{1}, m_{2}$ are two methods with group elements $g_{1}, g_{2}$, then the method formed by successive application of $m_{1}$ and $m_{2}$ has corresponding group element $g_{1} g_{2}$. If $g$ is a group element and $h$ is a real number, then $g^{(h)}$ denotes the group element such that for any $t \in T$ with order $r(t), g^{(h)}(t)=h^{r(t)} g(t)$. If $g$ corresponds to a method $m$ then $g^{(h)}$ corresponds to a method $m^{\prime}$ say, which is related to $m$ in such a way that the increment function for $m^{\prime}$ applied to the differential equation $y^{\prime}=f \circ y$ is identical to the increment function for $m$ applied to the equation $y^{\prime}=h f \circ y$. In particular, if $h$ is an integer, then $p^{(h)}=p^{h}$ (where the last exponent is the group-theoretic power).

We now introduce some further terminology.

Definition 5.1. The derivative $g^{\prime}$ of $g \in G$ is defined by the recurrence

$$
\begin{aligned}
g^{\prime}(\tau) & =1, \\
g^{\prime}(t u) & =g^{\prime}(t) g(u), \quad t, u \in T .
\end{aligned}
$$

From this point onward, we regard $G$ as a linear space over the real field R. If $g_{1}$, $g_{2} \in G, c_{1}, c_{2} \in \mathrm{R}$ then $c_{1} g_{1}+c_{2} g_{2}$ is defined by

$$
\left(c_{1} g_{1}+c_{2} g_{2}\right)(t)=c_{1} g_{1}(t)+c_{2} g_{2}(t)
$$

for all $t \in T$.

LemMA 5.2. For $\alpha, \beta, \gamma, \beta_{1}, \beta_{2}, \cdots, \beta_{n} \in G$ and $c, c_{1}, c_{2}, \cdots, c_{n} \in \mathbf{R}$ the following relations hold:

$$
\begin{aligned}
\alpha(c \beta) & =c \alpha \beta+(1-c) \alpha, \\
\alpha(\beta+\gamma) & =\alpha \beta+\alpha \gamma-\alpha, \\
\alpha \sum_{i=1}^{n} c_{i} \beta_{i} & =\sum_{i=1}^{n} c_{i} \alpha \beta_{i}+\left(1-\sum_{i=1}^{n} c_{i}\right) \alpha, \\
\alpha \beta^{\prime} & =\alpha+(\alpha \beta)^{\prime} .
\end{aligned}
$$

Proof. To prove (5.1), we make use of the function $\lambda$ introduced in [12]. We have, for $t \in T$, 


$$
\begin{aligned}
(\alpha(c \beta))(t) & =\alpha(t)+\lambda(\alpha, t)(c \beta) \\
& =\alpha(t)+c \lambda(\alpha, t)(\beta) \\
& =c(\alpha(t)+\lambda(\alpha, t)(\beta))+(1-c) \alpha(t) \\
& =c(\alpha \beta)(t)+(1-c) \alpha(t) .
\end{aligned}
$$

Similarly, to prove (5.2),

$$
\begin{aligned}
\alpha(\beta+\gamma)(t) & =\alpha(t)+\lambda(\alpha, t)(\beta+\gamma) \\
& =\alpha(t)+\lambda(\alpha, t)(\beta)+\alpha(t)+\lambda(\alpha, t)(\gamma)-\alpha(t) \\
& =(\alpha \beta)(t)+(\alpha \gamma)(t)-\alpha(t) .
\end{aligned}
$$

Making use of (5.1), we see that (5.3) holds with $n=1$. We now use (5.1) and (5.2) to complete the proof of (5.3) by induction. We have, assuming the result holds with $n$ replaced by $n-1$,

$$
\begin{aligned}
\alpha \sum_{i=1}^{n} c_{i} \beta_{i} & =\alpha\left(\sum_{i=1}^{n-1} c_{i} \beta_{i}+c_{n} \beta_{n}\right)=\alpha \sum_{i=1}^{n-1} c_{i} \beta_{i}+\alpha\left(c_{n} \beta_{n}\right)-\alpha \\
& =\sum_{i=1}^{n-1} c_{i}\left(\alpha \beta_{i}\right)+\left(1-\sum_{i=1}^{n-1} c_{i}\right) \alpha+c_{n}\left(\alpha \beta_{n}\right)+\left(1-c_{n}\right) \alpha-\alpha \\
& =\sum_{i=1}^{n} c_{i} \alpha \beta_{i}+\left(1-\sum_{i=1}^{n} c_{i}\right) \alpha .
\end{aligned}
$$

Finally, we note that (5.4) is equivalent to

$$
\left(\alpha \beta^{\prime}\right)(t)=\alpha(t)+(\alpha \beta)^{\prime}(t)
$$

for all $t \in T$ and this will now be proved by recursion. For $t=\tau$, the result is clear. We now assume (5.5) holds when $t=u$ and when $t=v$ and prove it when $t=u v$. Let $\lambda(\alpha, u)=\sum_{w \in T} c(w) \hat{w}, \lambda(\alpha, v)=\sum_{x \in T} d(x) \hat{x}$ where $c, d$ map all but a finite set of trees to 0 . Using results proved in [12], we find

$$
\begin{aligned}
\left(\alpha \beta^{\prime}\right)(u v) & =\alpha(u v)+\lambda(\alpha, u v)\left(\beta^{\prime}\right) \\
& =\alpha(u v)+(\lambda(\alpha, u) \lambda(\alpha, v))\left(\beta^{\prime}\right)+\alpha(v) \lambda(\alpha, u)\left(\beta^{\prime}\right) \\
& =\alpha(u v)+\sum_{w, x \in T} c(w) d(x) \beta^{\prime}(w x)+\alpha(v) \lambda(\alpha, u)\left(\beta^{\prime}\right) \\
& =\alpha(u v)+\sum_{w, x \in T} c(w) d(x) \beta^{\prime}(w) \beta(x)+\alpha(v) \lambda(\alpha, u)\left(\beta^{\prime}\right) \\
& =\alpha(u v)+\lambda(\alpha, u)\left(\beta^{\prime}\right)(\alpha(v)+\lambda(\alpha, v)(\beta)) \\
& =\alpha(u v)+\left(\left(\alpha \beta^{\prime}\right)(u)-\alpha(u)\right)(\alpha \beta)(v) \\
& =\alpha(u v)+(\alpha \beta)^{\prime}(u)(\alpha \beta)(v) \\
& =\alpha(u v)+(\alpha \beta)^{\prime}(u v) .
\end{aligned}
$$

This completes the proof of (5.5) and therefore of (5.4).

Let the Runge-Kutta methods $r_{1}, r_{2}, \cdots, r_{N}$ have group elements $g_{1}, g_{2}, \cdots, g_{N}$ associated with them when $h=1$. Thus, for a general stepsize $h$, the group elements 
are $g_{1}^{(h)}, g_{2}^{(h)}, \cdots, g_{N}^{(h)}$. We wish to study the group elements associated with $\bar{r}_{1}$, $\bar{r}_{2}, \cdots, \bar{r}_{N}$ given by (3.1).

THEOREM 5.3. The group elements associated with $\bar{r}_{1}, \bar{r}_{2}, \cdots, \bar{r}_{N}$ are given by $\overline{\boldsymbol{g}}_{1}^{(h)}, \overline{\boldsymbol{g}}_{2}^{(h)}, \cdots, \overline{\mathrm{g}}_{N}^{(h)}$ where

$$
\bar{g}_{i}=\sum_{i=1}^{N} a_{i} g_{i}+\sum_{i=1}^{N} b_{i} \bar{g}_{i}^{\prime}, \quad i=1,2, \cdots, N .
$$

Proof. We first note that (5.6) does indeed define $\bar{g}_{i}(t)$ for $i=1,2, \cdots, N$ and all $t \in T$, since in the equation

$$
\bar{g}_{i}(t)=\sum_{i=1}^{N} a_{i j} g_{j}(t)+\sum_{j=1}^{N} b_{i} \bar{g}_{i}^{\prime}(t), \quad i=1,2, \cdots, N,
$$

$\bar{g}_{j}^{\prime}(t)$ on the right-hand side can be written as the product $\bar{g}_{i}\left(t_{1}\right) \bar{g}_{i}\left(t_{2}\right) \cdots \bar{g}_{i}\left(t_{s}\right)$ say, where $t_{1}, t_{2}, \cdots, t_{s}$ have lower orders than $t$.

By absorbing $h$ into the function $f$, we can, without loss of generality, restrict ourselves to the case $h=1$ so that $g_{i}^{(h)}=g_{i}$ and $\bar{g}_{i}^{(h)}=\bar{g}_{i}$ for $i=1,2, \cdots, N$. Suppose the Runge-Kutta method $r_{i}(i=1,2, \cdots, N)$ is defined by $\left(S_{i}, c_{i}, s_{i}\right)$ where $S_{i}$ is a (finite) set, $c_{i}$ is a linear operator on the space of real-valued functions on $S_{i}$ and $s_{i}$ is a particular member of $S_{i}$. The result computed by the method $r_{i}$, starting from an initial value $z \in X$, is $y\left(s_{i}\right)$ where, for all $s \in S_{i}$,

$$
y(s)=z+\left(c_{i} \times(f \circ y)\right)(s) .
$$

Suppose, without loss of generality, that $S_{1}, S_{2}, \cdots, S_{N}$ are disjoint and that none of them contain any of the numbers $1,2, \cdots, N$. Form a new set $S=S_{1} \cup S_{2} \cup \ldots$ $\cup S_{N} \cup\{1,2, \cdots, N\}$ and define a function $y: S \rightarrow X$ satisfying

$$
y(s)=z+(c \times(f \circ y))(s)
$$

for all $s \in S$, where $c: B(S) \rightarrow B(S)$ is defined by $c(x) \mid S_{i}=c_{i}\left(x \mid S_{i}\right)$ for $i=1,2, \cdots, N$ and

$$
c(x)(i)=\sum_{i=1}^{N} a_{i j} c_{i}\left(x \mid S_{i}\right)\left(s_{i}\right)+\sum_{i=1}^{N} b_{i} x(j), \quad i=1,2, \cdots, N .
$$

Note that (5.8) defines $y$ for all $f$ with sufficiently small Lipschitz constant, and that $y\left(s_{i}\right)=r_{i}(1)(z), y(i)=\bar{r}_{i}(1)(z)$ for all $i=1,2, \cdots, N$. Let the functions $\mu, \nu$ be defined for $S$ and $c$ as described in [12] and, for $i=1,2, \cdots, N$, let $\mu_{i}, \nu_{i}$ be the corresponding functions computed for $S_{i}, c_{i}$, so that for all $t \in T, \mu_{i}(t)=\mu(t) \mid S_{i}$ and $\nu_{i}(t)=\nu(t) \mid S_{i}$. Also, for all $t \in T$ and all $i=1,2, \cdots, N, \nu(t)\left(s_{i}\right)=g_{i}(t)$, $\nu(t)(i)=\bar{g}_{i}(t)$, and $\mu(t)(i)=\bar{g}_{i}^{\prime}(t)$. We now use the relation $\nu(t)=c(\mu(t))$ to compute, for $i=1,2, \cdots, N$,

$$
\begin{aligned}
\bar{g}_{i}(t) & =\nu(t)(i)=c(\mu(t))(i) \\
& =\sum_{i=1}^{N} a_{i} c_{i}\left(\mu(t) \mid S_{i}\right)\left(s_{i}\right)+\sum_{i=1}^{N} b_{i} \mu(t)(j)=\sum_{i=1}^{N} a_{i} c_{j}\left(\mu_{i}(t)\right)\left(s_{i}\right)+\sum_{i=1}^{N} b_{i j} \mu(t)(j) \\
& =\sum_{i=1}^{N} a_{i} \nu_{i}(t)\left(s_{i}\right)+\sum_{j=1}^{N} b_{i j} \mu(t)(j)=\sum_{i=1}^{N} a_{i} g_{i}(t)+\sum_{i=1}^{N} b_{i j} \bar{g}_{i}^{\prime}(t) .
\end{aligned}
$$

Hence, (5.6) holds. 
We now use the result of Theorem 5.3 to express a condition for order. Let $T_{a}$ denote the set of trees with up to $q$ nodes.

THEOREM 5.4. The method $(A, B)$ is of order $q$ iff there exist $g_{1}, g_{2}, \cdots, g_{N} \in G$ such that, for all $t \in T_{a}$ and $i=1,2, \cdots, N$,

$$
\left(p g_{i}\right)(t)=\sum_{j=1}^{N} a_{i j} g_{j}(t)+\sum_{j=1}^{N} b_{i j}\left(p g_{i}\right)^{\prime}(t) .
$$

Proof. Given Runge-Kutta methods $r_{1}, r_{2}, \cdots, r_{N}$, let $g_{1}^{(h)}, g_{2}^{(h)}, \cdots, g_{N}^{(h)}$ denote the group elements associated with $r_{1}(h), r_{2}(h), \cdots, r_{N}(h)$. By Theorem 5.3, the group elements associated with $\bar{r}_{1}(h), \bar{r}_{2}(h), \cdots, \bar{r}_{N}(h)$ are $\bar{g}_{1}^{(h)}, \bar{g}_{2}^{(h)}, \cdots, \bar{g}_{N}^{(h)}$ where $\bar{g}_{1}$, $\bar{g}_{2}, \cdots, \bar{g}_{N}$ are given by (5.6). However, the group element associated with the operation that takes $z \in X$ to $r_{i}(h)\left(P_{h}(z)\right)$ is $p^{(h)} g_{i}^{(h)}=\left(p g_{i}\right)^{(h)}$. Hence, (5.9) will hold iff $\left(p g_{i}\right)(t)=\bar{g}_{s}(t)$ for $i=1,2, \cdots, N$ and all $t \in T_{a}$. This holds iff, for each $i=1,2, \cdots, N$, the Runge-Kutta method which, for stepsize $h$, takes $z \in X$ to $\left(r_{i}(h)^{-1} \circ \bar{r}_{i}(h)\right)(z)$ is of order $q$. This is equivalent to (3.2).

COROLlaRY 5.5. The method $(A, B)$ is of order $q$ iff there exist $g_{1}, g_{2}, \cdots, g_{N} \in G$ such that, for all $t \in T_{a}$ and $i=1,2, \cdots, N$,

$$
g_{i}(t)=\sum_{j=1}^{N} a_{i j}\left(p^{-1} g_{i}\right)(t)+\sum_{j=1}^{N} b_{i} g_{i}^{\prime}(t)
$$

Proof. This result follows directly from Theorem 5.4 by substituting $p^{-1} g_{i}$ for $g_{i}(i=1,2, \cdots, N)$. However, we will give an alternative proof based on the grouptheoretic interpretations of (5.9) and (5.10). Let $G_{a}$ denote the normal subgroup of $G$ such that, if $g \in G_{a}$ and $t \in T_{q}$, then $g(t)=0$. We will prove that

$$
g_{i}^{-1} p^{-1}\left(\sum_{i=1}^{N} a_{i j} g_{j}+\sum_{i=1}^{N} b_{i j}\left(p g_{j}\right)^{\prime}\right)=g_{i}^{-1}\left(\sum_{i=1}^{N} a_{i j}\left(p^{-1} g_{i}\right)+\sum_{i=1}^{N} b_{i j} g_{i}^{\prime}\right) \text {, }
$$

which will establish the result since (5.9) is equivalent to the statement that the lefthand side of (5.11) is in $G_{a}$ and (5.10) is equivalent to the statement that the right-hand side of (5.11) is in $G_{q}$. We have, using Lemma 5.2 and the consistency of $(A, B)$,

$$
\begin{gathered}
g_{i}^{-1} p^{-1}\left(\sum_{i=1}^{N} a_{i j} g_{i}+\sum_{i=1}^{N} b_{i j}\left(p g_{i}\right)^{\prime}\right) \\
=g_{i}^{-1}\left(\sum_{j=1}^{N} a_{i j}\left(p^{-1} g_{j}\right)+\sum_{i=1}^{N} b_{i j} p^{-1}\left(p g_{i}\right)^{\prime}+\left(1-\sum_{i=1}^{N} a_{i i}-\sum_{j=1}^{N} b_{i i}\right) p^{-1}\right) \\
=g_{i}^{-1}\left(\sum_{i=1}^{N} a_{i j}\left(p^{-1} g_{i}\right)+\sum_{i=1}^{N} b_{i j} g_{i}^{\prime}\right) \cdot \square
\end{gathered}
$$

In the next corollary, which we state without proof, we consider a method $(A, B, C)$ in which (2.2) is replaced by

$$
Y^{(n)}=[A] Y^{(n-1)}+h[B] F\left(Y^{(n)}\right)+\dot{h}[C] F\left(Y^{(n-1)}\right) .
$$

Corollary 5.6. The method $(A, B, C)$ is of order $q$ iff there exist $g_{1}, g_{2}, \cdots, g_{N}$ $\in G$ such that, for all $t \in T_{a}$ and $i=1,2, \cdots, N$,

$$
\left(p g_{i}\right)(t)=\sum_{i=1}^{N} a_{i j} g_{j}(t)+\sum_{i=1}^{N} b_{i i}\left(p g_{i}\right)^{\prime}(t)+\sum_{i=1}^{N} c_{i j} g_{i}^{\prime}(t)
$$


TABLE 6.1

\begin{tabular}{|c|c|c|c|c|c|c|}
\hline t & $\tau$ & $\tau$ & $\tau \tau . \tau$ & T.tT & 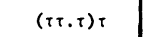 & \\
\hline$g_{1}^{\prime}(t)$ & 1 & 0 & 0 & $\frac{1}{2}\left(b_{42}+b_{43}-1\right)$ & 0 & 0 \\
\hline$g_{2}^{\prime}(t)$ & 1 & $\frac{1}{2}$ & $\frac{1}{4}$ & 0 & $\frac{1}{8}$ & 0 \\
\hline$s_{3}^{\prime}(t)$ & 1 & $\frac{1}{2}$ & $\frac{1}{4}$ & $\frac{1}{2} b_{32}$ & $\frac{1}{8}$ & $\frac{1}{4} b_{32}$ \\
\hline $8_{4}^{\prime}(t)$ & 1 & 1 & 1 & $\frac{1}{2}\left(b_{42}+b_{43}\right)$ & 1 & $\frac{1}{2}\left(b_{42}+b_{43}\right)$ \\
\hline $8_{1}(t)$ & 0 & $\frac{1}{2}\left(b_{42}+b_{43}-1\right)$ & $\frac{2}{3}-\frac{3}{4}\left(b_{42}+b 43\right)$ & $-\frac{1}{6}+\frac{1}{2} b_{43} b_{32}+\frac{1}{2}\left(b_{42}+b_{43}\right)\left(1-b_{42}-b_{43}\right)$ & $\frac{7}{8}\left(\mathrm{~b}_{42}+\mathrm{b}_{43}\right)-\frac{3}{4}$ & $\frac{1}{2}\left(b_{42}+b_{43}-\frac{1}{2}\right)^{2}-\frac{1}{4} b_{43} b_{32}$ \\
\hline$g_{2}(t)$ & $\frac{1}{2}$ & 0 & 0 & $\frac{1}{4}\left(b_{42}+b_{43}-1\right)$ & 0 & 0 \\
\hline$z_{3}(t)$ & $\frac{1}{2}$ & $\frac{1}{2} b_{32}$ & $\frac{1}{4} b_{32}$ & $\frac{1}{2}\left(\frac{1}{2}-b_{32}\right)\left(b_{42}+b_{43}-1\right)$ & $\frac{1}{8} b_{32}$ & 0 \\
\hline $8_{4}(t)$ & 1 & $\frac{1}{2}\left(b_{42}+b_{43}\right)$ & $\frac{1}{4}\left(b_{42}+b_{43}\right)$ & $-\frac{1}{2}\left(1-b_{42}-b_{43}\right)^{2}+\frac{1}{2} b_{43} b_{32}$ & $\frac{1}{8}\left(b_{42}+b_{43}\right)$ & $\frac{1}{4} b_{43^{b}} b_{32}$ \\
\hline$g_{5}(t)$ & 1 & $\frac{1}{2}$ & $\frac{1}{3}$ & $--\frac{1}{12}+\frac{1}{2} b_{53} b_{32}+\frac{1}{6}\left(b_{42}+b_{43}\right)$ & $\frac{1}{4}$ & $\frac{1}{4} b_{53} b_{32}+-\frac{1}{12}\left(b_{42}+b_{43}\right)$ \\
\hline$p(t)$ & 1 & $\frac{1}{2}$ & $\frac{1}{3}$ & $\frac{1}{6}$ & $\frac{1}{4}$ & $\frac{1}{8}$ \\
\hline
\end{tabular}

6. An Example. By contrast with the classical Runge-Kutta method $(A, B)$ where

$$
A=\left[\begin{array}{lllll}
0 & 0 & 0 & 0 & 1 \\
0 & 0 & 0 & 0 & 1 \\
0 & 0 & 0 & 0 & 1 \\
0 & 0 & 0 & 0 & 1 \\
0 & 0 & 0 & 0 & 1
\end{array}\right], \quad B=\left[\begin{array}{ccccc}
0 & 0 & 0 & 0 & 0 \\
\frac{1}{2} & 0 & 0 & 0 & 0 \\
0 & \frac{1}{2} & 0 & 0 & 0 \\
0 & 0 & 1 & 0 & 0 \\
\frac{1}{6} & \frac{1}{3} & \frac{1}{3} & \frac{1}{6} & 0
\end{array}\right]
$$

we consider a method $(A, B)$ where

$$
A=\left[\begin{array}{lllll}
0 & 0 & 0 & 1 & 0 \\
0 & 0 & 0 & 0 & 1 \\
0 & 0 & 0 & 0 & 1 \\
0 & 0 & 0 & 0 & 1 \\
0 & 0 & 0 & 0 & 1
\end{array}\right], \quad B=\left[\begin{array}{ccccc}
0 & 0 & 0 & 0 & 0 \\
\frac{1}{2} & 0 & 0 & 0 & 0 \\
\frac{1}{2}-b_{32} & b_{32} & 0 & 0 & 0 \\
\frac{1}{2}-b_{42}-b_{43} & b_{42} & b_{43} & 0 & 0 \\
\frac{1}{6} & \frac{2}{3}-b_{53} & b_{53} & \frac{1}{6} & 0
\end{array}\right]
$$

and $b_{32}, b_{42}, b_{43}, b_{53}$ are to be chosen. The advantage the method (6.2) would have over (6.1) is that it requires three rather than four derivative calculations per step. This 
TABLE 6.1 (continued)

\begin{tabular}{|c|c|}
\hline$\tau(\tau, \tau)$ & $\tau(\tau . \tau T)$ \\
\hline$\frac{2}{3}-\frac{3}{4}\left(b_{42}+b_{43}\right)$ & $-\frac{1}{6}+\frac{1}{2} b_{43} b_{32}+\frac{1}{2}\left(b_{42}+b_{43}\right)\left(1-b_{42} 2^{-b} b_{43}\right)$ \\
\hline 0 & $\frac{1}{4}\left(b_{42}+b_{43}-1\right)$ \\
\hline$\frac{1}{4} b_{32}$ & $\frac{1}{2}\left(\frac{1}{2}-b_{32}\right)\left(b_{42}+b_{43}-1\right)$ \\
\hline$\frac{1}{4}\left(b_{42}+b 43\right)$ & $-\frac{1}{2}\left(1-b_{42}-b_{43}\right)^{2}+\frac{1}{2} b_{43} b_{32}$ \\
\hline$\frac{12-35}{12}\left(b_{12}+2^{+b}\right)+\frac{2}{4}\left(b_{42}+b_{43}\right)^{2}-\frac{3}{4} b_{43} b_{32}$ & $b_{43} b_{32}\left(\frac{1}{2}-b_{42}-b_{43}\right)+\frac{5}{24}-\frac{1}{3}\left(b_{42}+b_{43}\right)-\frac{1}{2}\left(b_{42}+b_{43}\right)^{2}\left(\frac{1}{2}-b_{42}-b_{43}\right)$ \\
\hline$\frac{1}{3}-\frac{3}{8}\left(b_{42}+b_{43}\right)$ & $\left.-\frac{1+4 b_{6}}{12} 43^{b} 32_{4}+\frac{1}{4} b_{42}+b_{43}\right)\left(1-b_{42}-b_{43}\right)$ \\
\hline$\left(\frac{1}{2}-b_{32}\right)\left(\frac{2}{3}-\frac{3}{4}\left(b_{42}+b_{43}\right)\right)$ & $\left(\frac{1}{2}-b_{32}\right)\left(-\frac{1}{6}+\frac{1}{2} b_{43} b_{32}\right)+\left(1-b_{42}-b_{43}\right)\left(\frac{1}{4}\left(b_{42}+b_{43}\right)-b_{32}\left(\frac{1}{4} \frac{1}{2}\left(b_{42}+b_{43}\right)\right)\right)$ \\
\hline$\frac{2}{3}-\frac{17}{12}\left(b_{42}+b_{43}\right)+\frac{3}{4}\left(b_{42}+b_{43}\right)^{2}+\frac{1 b}{4} 43_{32}$ & $\left(1-b_{42}-b_{43}\right)\left(-\frac{1}{6}+\frac{1}{4}\left(b_{42}+b_{43}\right)-\frac{1}{2}\left(b_{42}+b_{43}\right)^{2}+b_{43} b_{32}\right)$ \\
\hline$\frac{1}{9}-\frac{1}{12}\left(b_{42}+b_{43}\right)+\frac{1}{4} b_{53} b_{32}$ & $-\frac{5,+5}{18}\left(b_{42}+2_{43}\right) \frac{1}{6}\left(b_{42}+b_{43}\right)^{2}+\frac{1}{6} b_{43} b_{32}+\frac{1}{2} b_{53} b_{32}\left(1-b_{42} 2_{43}^{-b}\right)$ \\
\hline$\frac{1}{12}$ & $\frac{1}{24}$ \\
\hline
\end{tabular}

is because $f\left(y_{1}^{(n)}\right)$ is identical to $f\left(y_{4}^{(n-1)}\right)$ and need not be recomputed and because $f\left(y_{5}^{(n)}\right)$ is not made use of and therefore need not be computed at all.

What we shall do is to choose $b_{32}, b_{42}, b_{43}, b_{53}$, so that the method (6.2) is of order 4 relative to $r_{1}, r_{2}, r_{3}, r_{4}, r_{5}$ where $r_{5}$ is the trivial Runge-Kutta method defined by $r_{5}(h)(z)=z$. This will mean that the method will not require any special finishing procedure. Let $p^{-1} g_{1}, p^{-1} g_{2}, p^{-1} g_{3}, p^{-1} g_{4}, p^{-1} g_{5}$ (where $g_{5}=p$ ) denote the group elements corresponding to $r_{1}(1), r_{2}(1), r_{3}(1), r_{4}(1), r_{5}(1)$ so that the conditions for the method to be of order 4 are that, for $t \in T_{4}$,

$$
\begin{aligned}
g_{1}(t) & =\left(p^{-1} g_{4}\right)(t), \\
g_{2}(t) & =\frac{1}{2} g_{1}^{\prime}(t), \\
g_{3}(t) & =\left(\frac{1}{2}-b_{32}\right) g_{1}^{\prime}(t)+b_{32} g_{2}^{\prime}(t), \\
g_{4}(t) & =\left(1-b_{42}-b_{43}\right) g_{1}^{\prime}(t)+b_{42} g_{2}^{\prime}(t)+b_{43} g_{3}^{\prime}(t), \\
p(t)=g_{5}(t) & =\frac{1}{6} g_{1}^{\prime}(t)+\left(\frac{2}{3}-b_{53}\right) g_{2}^{\prime}(t)+b_{53} g_{3}^{\prime}(t)+\frac{1}{6} g_{4}^{\prime}(t) .
\end{aligned}
$$

To write these as polynomial conditions on $b_{32}, b_{42}, b_{43}, b_{53}$, we substitute in turn $t=\tau, \tau \tau, \tau \tau \cdot \tau, \tau \cdot \tau \tau,(\tau \tau \cdot \tau) \tau, \tau \tau \cdot \tau \tau, \tau(\tau \tau \cdot \tau), \tau(\tau \cdot \tau \tau)$ into (6.3)-(6.7). From (6.4), (6.5), (6.6), we find, for each $t$, the values of $g_{2}(t), g_{3}(t), g_{4}(t)$; from (6.3), we find the value of $g_{1}(t)$; and, from (6.7), we obtain a condition on $b_{32}, \cdots, b_{53}$. Because of the form chosen for $B$, the equations for $t=\tau, \tau \tau, \tau \tau \cdot \tau,(\tau \tau \cdot \tau) \tau$ will be automatically satisfied. However, for completeness, the computations are shown for all the 8 members of $T_{4}$ in Table 6.1. 
Equating $g_{5}(t)$ with $p(t)$ in the cases $t=\tau \cdot \tau \tau, \tau \tau \cdot \tau \tau, \tau(\tau \tau \cdot \tau)$ and $\tau(\tau \cdot \tau \tau)$, we obtain the following conditions

$$
\begin{gathered}
\frac{1}{6}\left(b_{42}+b_{43}\right)-\frac{1}{12}+\frac{1}{2} b_{53} b_{32}=\frac{1}{6}, \\
\frac{1}{12}\left(b_{42}+b_{43}\right)+\frac{1}{4} b_{53} b_{32}=\frac{1}{8}, \\
\frac{1}{9}-\frac{1}{12}\left(b_{42}+b_{43}\right)+\frac{1}{4} b_{53} b_{32}=\frac{1}{12} \\
-\frac{5}{18}+\frac{5}{12}\left(b_{42}+b_{43}\right)-\frac{1}{6}\left(b_{42}+b_{43}\right)^{2} \\
+\frac{1}{6} b_{43} b_{32}+\frac{1}{2} b_{53} b_{32}\left(1-b_{42}-b_{43}\right)=\frac{1}{24} .
\end{gathered}
$$

Equations (6.8), (6.9), (6.10) are satisfied if and only if

$$
\begin{aligned}
b_{42}+b_{43} & =11 / 12, \\
b_{53} b_{32} & =7 / 36,
\end{aligned}
$$

and, with these values, (6.11) simplifies to

$$
b_{43} b_{32}=5 / 12 \text {. }
$$

The general solution to (6.12), (6.13), (6.14) is

$$
b_{32}=\theta, \quad b_{42}=11 / 12-5 / 12 \theta, \quad b_{43}=5 / 12 \theta, \quad b_{53}=7 / 36 \theta
$$

where $\theta$ is any nonzero number. There do not seem to be compelling reasons for choosing any particular value for $\theta$; an analysis of the region of absolute stability, for example, shows this to be independent of $\theta$. Hence, we select $\theta=\frac{1}{2}$ on grounds of simplicity. The method (6.2) becomes in this case

$$
A=\left[\begin{array}{ccccc}
0 & 0 & 0 & 1 & 0 \\
0 & 0 & 0 & 0 & 1 \\
0 & 0 & 0 & 0 & 1 \\
0 & 0 & 0 & 0 & 1 \\
0 & 0 & 0 & 0 & 1
\end{array}\right], \quad B=\left[\begin{array}{ccccc}
0 & 0 & 0 & 0 & 0 \\
\frac{1}{2} & 0 & 0 & 0 & 0 \\
0 & \frac{1}{2} & 0 & 0 & 0 \\
\frac{1}{12} & \frac{1}{12} & \frac{5}{6} & 0 & 0 \\
\frac{1}{6} & \frac{5}{18} & \frac{7}{18} & \frac{1}{6} & 0
\end{array}\right] \text {. }
$$

To use this method in practice, we could find Runge-Kutta methods $r_{1}, r_{2}, r_{3}, r_{4}$ which satisfy the requirements that $r_{i}(1)$ has group element lying in $p^{-1} g_{i} G_{4}$ and use these methods to compute starting values for $Y_{i}^{(0)}(i=1,2,3,4)$. However, since only $h f\left(Y_{4}^{(0)}\right)$ and $Y_{5}^{(0)}$ are used in subsequent steps, $r_{1}, r_{2}, r_{3}$ can be omitted from consideration and $Y_{4}^{(0)}$ need be computed only to third-order accuracy. The starting procedure that will now be suggested takes the result forward one step (so that we may think of $Y_{4}^{(1)}, Y_{5}^{(1)}$ as being the starting values computed) by the classical fourthorder method (6.1) and then computes $Y_{4}^{(1)}$ by using an increment function that requires no further derivative calculations. If $Y_{1}, Y_{2}, Y_{3}, Y_{4}, Y_{5}$ are the approximations computed using (6.1) with $Y_{1}$ equal to a given initial value $y\left(x_{0}\right)$, we compute $Y_{4}^{(1)}, Y_{5}^{(1)}$ as follows

$$
\begin{aligned}
& Y_{2}=Y_{1}+\frac{1}{2} h f\left(Y_{1}\right), \\
& Y_{3}=Y_{1}+\frac{1}{2} h f\left(Y_{2}\right),
\end{aligned}
$$




$$
\begin{aligned}
Y_{4} & =Y_{1}+h f\left(Y_{3}\right), \\
Y_{5}^{(1)} & =Y_{5}=Y_{1}+\frac{1}{6} h\left(f\left(Y_{1}\right)+2 f\left(Y_{2}\right)+2 f\left(Y_{3}\right)+f\left(Y_{4}\right)\right), \\
Y_{4}^{(1)} & =Y_{1}+h\left(c_{1} f\left(Y_{1}\right)+c_{2} f\left(Y_{2}\right)+c_{3} f\left(Y_{3}\right)+c_{4} f\left(Y_{4}\right)\right),
\end{aligned}
$$

where $c_{1}, c_{2}, c_{3}, c_{4}$ are real numbers. If $g^{(h)}$ is the group element associated with $Y_{4}^{(1)}$ computed this way, we find

$$
\begin{aligned}
g(\tau) & =c_{1}+c_{2}+c_{3}+c_{4}, \\
g(\tau \tau) & =\frac{1}{2}\left(c_{2}+c_{3}\right)+c_{4}, \\
g(\tau \tau \cdot \tau) & =\frac{1}{4}\left(c_{2}+c_{3}\right)+c_{4}, \\
g(\tau \cdot \tau \tau) & =\frac{1}{4} c_{3}+\frac{1}{2} c_{4},
\end{aligned}
$$

so that, equating these to the appropriate $g_{4}(t)$ given in Table 6.1 , we obtain the values

$$
c_{1}=1 / 12, \quad c_{2}=7 / 72, \quad c_{3}=59 / 72, \quad c_{4}=0 .
$$

Although this new method is put forward to illustrate the definition of order rather than as a practical alternative to existing methods, it does seem appropriate to see how well it performs in at least one example. Accordingly, the four-dimensional system of equations $y_{i}^{\prime}(\xi)=y_{i+1}(\xi)(i=1,3), y_{i}^{\prime}(\xi)=-y_{i-1}(\xi) /\left(y_{1}(\xi)^{2}+y_{3}(\xi)^{2}\right)^{3 / 2}$ $(i=2,4)$ with $y(0)=(1,0,0,1)$ was integrated through $\nu$ steps with stepsize $h=\pi / 2 \nu$ and with $\nu=10,20,40,80$ using the classical fourth-order Runge-Kutta method and the method derived in this section. At each value of $\nu$, for each method, the value of $\nu^{4}\left(y(\pi / 2)-\tilde{y}_{v}\right)$ was printed out. For each method, this vector was almost independent of $\nu$ and had the values

Runge-Kutta: $(.03, .10, .10, .13)$, New Method: $(.22, .04, .05, .27)$.

Thus, the fourth-order error behaviour for the new method is experimentally confirmed in this example. Although a comparison of these error vectors favours the Runge-Kutta method, a comparison based on numbers of derivative calculations (rather than numbers of steps) would favour the new method.

7. Acknowledgments. This paper was prepared at the University of Dundee where the author was participating in the North British Symposium on Differential Equations. He expresses his thanks to the Science Research Council of Great Britain which sponsored the Symposium and to the University of Dundee which made its facilities available to him.

Department of Mathematics

University of Auckland

Auckland, New Zealand

1. G. DAHLQuist, "Stability and error bounds in the numerical integration of ordinary differential equations," Kungl. Tekn. Högsk. Handl. Stockholm, No. 130, 1959, 87 pp. MR 21 \#1706.

2. P. HenricI, Discrete Variable Methods in Ordinary Differential Equations, Wiley, New York, 1962. MR 24 \#B1772.

3. M. URABE, "Theory of errors in numerical integration of ordinary differential equations," J. Sci. Hiroshima Univ. Ser. A-I Math., v. 25, 1961, pp. 3-62. MR 25 \#759. 
4. H. ShintanI, "Approximate computation of errors in numerical integration of ordinary differential equations by one-step methods," J. Sci. Hiroshima Univ. Ser. A-I Math., v. 29, 1965, pp. 97-120. MR 31 \#6383.

5. M. Urabe, H. Yanagiwara \& Y. Shinohara, "Periodic solutions of van der Pol's equation with damping coefficient $\lambda=2 \sim 10$," J. Sci. Hiroshima Univ. Ser. A-1 Math., v. 23, 1960, pp. 325-366. MR 23 \#A1889.

6. W. B. GragG \& H. J. STETTER, "Generalized multistep predictor-corrector methods," J. Assoc. Comput. Mach., v. 11, 1964, pp. 188-209. MR 28 \#4680.

7. J. C. Butcher, "A modified multistep method for the numerical integration of ordinary differential equations," J. Assoc. Comput. Mach., v. 12, 1965, pp. 124-135. MR 31 \#2830.

8. C. W. GEAR, "Hybrid methods for initial value problems in ordinary differential equations," J. Soc. Indust. Appl. Math. Ser. B. Numer. Anal., v. 2, 1965, pp. 69-86 MR 31 \#3738.

9. J. C. BuTCHER, "A multistep generalization of Runge-Kutta methods with four or five stages,"J. Assoc Comput Mach., v. 14, 1967. pp. 84-99. MR 35 \#3896.

10. J. C. BUTCHER, "On the convergence of numerical solutions to ordinary differential equations," Math. Comp., v. 20, 1966, pp. 1-10. MR 32 \#6678.

11. J. C. Butcher, "The effective order of Range-Kutta methods," Conf. on Numerical Solution of Differential Equations (Dundee 1969), Springer, Berlin, 1969, pp. 133-139. MR 43 \#1432.

12. J. C. Butcher, "An algebraic theory of integration methods," Math. Comp., v. 26, 1972, pp. 79-106. 